Revista de Psicología Vol. 35 (2), 2017 (ISSN 0254-9247)

\title{
Diseño del cuestionario: Estrategias identitarias de académicos universitarios ante las reformas educativas
}

\author{
Elizabeth Zanatta Colín ${ }^{1}$, Teresa Ponce Dávalos², Sergio Luis García \\ Iturriaga $^{3}$, Claudia Angélica Sánchez Calderón ${ }^{4}$, José Luis Gama Vilchis ${ }^{5}$ \\ Universidad Autónoma del Estado de México
}

\begin{abstract}
Se diseñó un cuestionario que tiene la finalidad de comprender qué sucede con la identidad del académico, como elemento rector de su práctica docente, ante las reformas educativas que han generado un cambio constante en lo que se espera de su función. El cuestionario se aplicó a 228 académicos de diferentes disciplinas de una Universidad pública estatal con un modelo educativo centrado en competencias. Los datos fueron procesados considerando el análisis factorial y el alfa de Cronbach. Los hallazgos fueron congruentes con la estructura factorial hipotética, con un porcentaje de varianza aceptable (43\%), que explica cuatro factores de estrategias identitarias: resistente-difusa, atribuida, simulada y asumida. El instrumento reportó un adecuado nivel de consistencia interna (.94). El cuestionario cuenta con validez de constructo y consistencia interna.

Palabras clave: identidad docente, disposición docente, estrategias identitarias, construcción de instrumentos, praxis educativa.
\end{abstract}

1 Doctora en Educación por la Universidad Autónoma del Estado de Morelos. Profesor investigador de tiempo completo UAEMéx. Dirección: Isabel La Católica sur \# 906. Col Sn. Sebastián Toluca, México. C.P. 20150. Contacto: elizatt12@live.com

2 Maestra en Administración por Universidad Autónoma del Estado de México. Profesor investigador de tiempo completo UAEMéx. Dirección: Cedral del Centro 23, Col. La Crespa. Toluca, México. C.P. 50010. Contacto: tpd1210@hotmail.com

3 Maestro en Administración por Universidad Autónoma del Estado de México. Profesor Investigador de tiempo completo UAEMéx. Dirección: Cedral del Centro 23, Col. La Crespa. Toluca, México. C.P. 50010. Contacto: jefeslgi5512@hotmail.com

4 Doctora en Educación Instituto Superior de Ciencias de la Educación del Estado de México. Profesor investigador de tiempo completo UAEMéx. Dirección: Privada de Laureles lote 18 casa 18 Residencial Foresta. Colonia San Miguel Totocuitlapilco CP 52143 Municipio de Metepec. Contacto: clau_sc2014@hotmail.com

5 Maestro en Psicología por Universidad Autónoma del Estado de México. Profesor investigador de tiempo completo UAEMéx. Dirección: Pino Suarez sur No 2028. Col. Benito Juárez. Toluca, Estado de México, CP. 501910. Contacto: ari7203_2@yahoo.com.mx 
Questionnaire design: identity strategies in university academics in response to the educational reforms

We designed a questionnaire that aims to understand what happens to the identity of the teacher as a guiding element of its educative practice in response to the educational reforms -which have generated a constant change in what is expected from their role. The questionnaire was applied to 228 academics from different disciplines in a state public University with an educational model based on competencies. The data were processed by factor analysis and Cronbach's Alpha. The results were consistent with the hypothesized factor structure, obtaining an acceptable percentage of variance (43\%), which explains four factors of identity strategies: resistant-diffuse, attributed, simulated and assumed. The instrument reported an adequate level of internal consistency (.94). Therefore, the questionnaire has both construct validity and internal consistency.

Keywords: teacher identity, teaching disposition, identity strategies, instrument development, educative praxis.

\section{Desenho do questionário: estratégias identitárias dos académicos universitários frente às reformas educativas.}

Desenhou-se um questionário com a finalidade de compreender o que aconteceu com a identidade do acadêmico, como elemento principal da sua prática docente, mediante as reformas educativas as quais têm gerado mudanças no que se refere à sua função. O questionário foi aplicado a 228 acadêmicos de diferentes disciplinas numa universidade pública estadual, que possui um modelo educativo orientado às competências. A análises foi fatorial e Alpha de Cronbach. Os descobrimentos foram congruentes com a estrutura fatorial hipotética, com a obtenção da porcentagem duma variável aceitável (43\%), a qual explica quatro fatores de estratégias identitárias: resistente-difusa; atribuída; simulada e assumida. O instrumento reportou um adequado nível de consistência interna (94). O questionário conta com a validade do construto e da consistência interna.

Palavras-chave: identidade docente; disposição docente; estratégias identitárias, construção de instrumentos; praxis educativa. 
La educación superior se enfrenta a nuevos paradigmas respecto a su calidad y pertinencia. Diferentes organismos internacionales y nacionales han realizado un incesante flujo de reformas educativas que han generado nuevos modelos educativos y distintas modalidades de la práctica del docente. En las últimas décadas, el docente ha pasado de una función transmisora, a moldear, a acompañar para la construcción de conocimientos y a formar en competencias. Rosas (2015) afirma que el docente, en el escenario universitario, actualmente se desempeña como facilitador, gestor, moderador y coordinador de la adquisición de conocimientos. Estas distintas formas de comprender y demandar diversificaciones al rol del académico condicionan transformaciones en su significado sobre el ser docente. Caballero (2013) afirma que se ha ido transformando la profesión y que existen nuevas características que hoy definen el perfil profesional del profesorado universitario.

Las actuales demandas de las prácticas educativas requieren de un proceso de enculturación ${ }^{6}$ para evitar la falta de sentido en su actividad y sus principios. La formación docente implica un proceso de enculturación para favorecer la reconstrucción del sentido de identidad y dar significado a las nuevas modalidades de su práctica educativa. Por ello, es importante identificar y comprender las estrategias identitarias que realiza el docente para encontrar sentido ante demandas y cambios constantes de su función académica.

El tema de la identidad ha sido de gran interés para los investigadores mexicanos. En los últimos ańos se ha trabajado en relación a: tipos y procesos de construcción de la identidad personal, la identidad social y la identidad cultural. En torno a la identidad social,

6 Teóricos humanistas de la formación, tal es el caso de Yurén (2005), sostienen que la formación de los formadores comprende un proceso de enculturación que contribuya a la reconstrucción de su identidad. 
comprendida como sentido de pertenencia a un determinado grupo social o laboral, se ha problematizado respecto a las transformaciones de las identidades ante escenarios y demandas cambiantes originadas por la globalización. Para Erikson (1956), la crisis de identidades es generada por importantes cambios de referentes y valores que producen vacíos de significados. Uno de los espacios donde se han generado constantes innovaciones es en el significado del rol y su práctica docente. Condición que posibilita la presencia de este tipo de estrategia identitaria en los académicos.

Los trabajos de investigación sobre el tema de la identidad profesional del docente han girado en torno a los roles y la imagen social de la profesión como sustento de la identidad (Ducoing, 2005). Desde posiciones humanistas, la identidad profesional se relaciona con el proceso personal para identificarse, pensarse y comportarse como docente.

Harsch (1994) propone una construcción teórica de los elementos que configuran la identidad. La autora sostiene que la identidad profesional comprende la constitución de: un yo-individual, un yo-teórico (cogniciones), un yo-ético (valores) y un yo-empírico (prácticas). La explicación de la integración de estos elementos requiere de la conformación del cuerpo de conocimientos a través de la historia de la disciplina, las funciones profesionales y los principales campos de su aplicación, así como del proceso de incorporación de estos conocimientos, y funciones desde la singularidad de cada sujeto que se forma. Muńoz y Arvayo (2015) identificaron cuatro elementos que configuran la identidad docente: a) la implicación del profesor en el proceso enseñanza-aprendizaje, b) el compromiso social que representa ser profesor, c) las asociaciones afectivas que despierta la labor docente, y d) la formación pedagógica y disciplinar que debe caracterizar al profesor universitario. Dichos elementos comprenden cogniciones, prácticas, valores, afectos y normas compartidas por grupos de académicos de disciplinas particulares.

Desde un enfoque sociológico, se afirma que las transformaciones que se generan en la identidad son producto de condiciones sociales cambiantes generadas por la globalización. Autores como 
Camillieri $^{7}$ (1990) y Castells (1998) proponen que ante los cambios sociales el sujeto desarrolla estrategias "identitarias" para crear sentido ante cambios constantes que generan confusión.

La propuesta de estrategias identitarias de Camillieri (1990) explica las disposiciones de los migrantes ante la cultura de origen y la cultura en la que se introducen. Este autor propone que las disposiciones se abren en un abanico de tres posibilidades: identidades dependientes, identidades por distinción e identidades reaccionarias. Considera a las dependientes como identidades negativas en tanto que el sujeto se anula en su individualidad, interioriza lo negativo de su identidad y adopta una actitud sumisa y recogida. A través de esta estrategia, el migrante se asimila en la nueva cultura y rechaza la cultura de origen. En la identidad por distinción, el sujeto logra mantener su individualidad al marcar distancia entre las dos culturas y al evita interiorizar las atribuciones negativas acerca de su identidad de origen. Camillieri (1990) identifica un tercer grupo de estrategias identitarias: las identidades reaccionarias, que mantienen la identificación con su grupo de origen y rechazan los valores, prácticas y creencias de la nueva cultura.

Castells (1998) propone que el sujeto desarrolla estrategias identitarias ante los cambios suscitados por la modernidad, sin la necesidad de migrar a una nueva cultura. Para él, las estrategias identitarias obedecen a la necesidad de crear sentido ante pluralidades que generan tensión y contradicción. Además, las estrategias identitarias que propone ese autor, presentan cierta correspondencia con las propuestas por Camillieri (1990), como se puede observar en su denominación: identidades legitimadoras con las identidades dependientes, de resistencia con las reaccionarias y de proyecto con las identidades por distinción.

Desde la posición de estos autores, es posible recuperar premisas teóricas sobre los procesos de construcción de la identidad, los elementos que la configuran y las posibles estrategias identitarias que se asumen ante diversos factores sociopersonales. En la presente investigación

Camillieri considera que ante el cambio, el individuo realiza operaciones cognitivas que le permiten actuar sobre las construcciones simbólicas de sus identidades para modificarlas, mantenerlas o matizarlas confrontando o negociando ante una realidad nueva. 
se tomaron como punto de partida las aportaciones de estas teorías para explorar las estrategias identitarias que construyen los académicos ante los cambios de paradigma en su función, generados por políticas y reformas educativas que le demandan una forma de realizar su práctica docente.

Se partió de un análisis teórico que permitió delimitar los referentes conforme a los cuales se constituye cada estrategia, dando lugar a una determinada configuración identitaria ${ }^{8}$. Como resultado de esto, se identificaron diferentes estrategias de identidad ${ }^{9}$ que se organizan en posturas teóricas y un tipo de proyecto profesional que revela un determinado nivel de consolidación de la identidad pedagógica y profesional.

La construcción hipotética de la posible estructura factorial se delimitó con base en dichos autores (Camillieri, 1990 y Castells, 1998). La denominación propuesta guarda relación con las teorías de sustento: identidad atribuida (acorde a la identidad dependiente de Camillieri o legitimadora de Castells); el factor de identidad de resistencia (en sintonía con la identidad reaccionaria de Camillieri o de resistencia de Castells); el factor de identidad asumida (en congruencia con la identidad por distinción de Camillieri o la identidad de proyecto de Castells). Asimismo, a través de la observación, se detectaron otros dos tipos de disposiciones docentes: simular asumir las disposiciones del modelo educativo (practicas docentes ya señaladas por Díaz Barriga en 2005), así como la total confusión de referentes prácticas y valores (propuesta por Erikson en 1956) ante cambios sociales que generan vacíos. Dichas propuestas teóricas y la información recabada de la observación permitió integrar los factores de simulación y difusión de la identidad.

8 Se comprende por configuración identitaria la conjugación de diversos tipos de creencias y cogniciones, prácticas, valores y normas que permiten la constitución de cada tipo de estrategia identitaria. Es decir, los elementos que permiten la identificación y caracterización de la dimensión.

9 Mientras que la configuración identitaria permite la caracterización de la identidad, las estrategias de identidad (resistencia, difusa, atribuida, simulación y asumida) representan la diferenciación en la identidad de los sujetos, con base en las diferentes representaciones de cada uno de los elemento de la configuración. 
Se previó que las estrategias se estructuren en las configuraciones propuestas por Castells (1998) quien señala que cada estrategia comprende diferentes tipos de creencias, prácticas, valores y normas. De igual forma, se presume que los académicos universitarios presentan indicadores de crisis de identidad en modalidad de confusión sobre su rol, simulación o asimilación irreflexiva de las demandas, mismas que dificultan su praxis educativa y reflejan la ausencia de un conjunto de disposiciones del claustro docente para contribuir a la adquisición de competencias y a la formación de profesionales.

Crisis y tensiones favorecidas por la instrumentación de un dispositivo técnico y funcional que dificulta la formación de un "Ethos" docente, un conjunto de disposiciones sustentadas en la construcción de una dimensión ética ${ }^{10}$ que promueva la reconstrucción de la identidad docente ante nuevos paradigmas y favorezca el asumir de manera comprometida los nuevos roles y modalidades que le demanda el enfoque pedagógico del modelo educativo vigente. La formación del "Ethos" requiere de un proceso de enculturación que favorezca la reconstrucción de la identidad del docente.

\section{Método}

\section{Participantes}

Se aplicaron cuestionarios a académicos de tiempo completo y de asignatura de la institución en estudio, seleccionados a través de un muestreo por cuota. Las características de la muestra de 228 académicos que participaron en el estudio fueron las siguientes: en cuanto a la variable edad el $34.6 \%$ (79) se encontraron en un rango de 28 a 40 años, el $37.3 \%$ (85) en un rango de 41 a 50 años y el restante $28.1 \%$

10 López (2003) sostiene que actualmente los académicos presentan una disminución de compromiso por la incertidumbre que les generan los cambios en los saberes de enseńanza que implican una valoración de lo técnico, la primacía de los valores económicos y la incorporación como moda de ciertos paradigmas pedagógicos. 
(64) más de 50 años. Con respecto al sexo: el 53.5\% (122) pertenecen al sexo masculino y el $46.5 \%$ (106) corresponden al sexo femenino.

\section{Instrumento}

Se diseñó un cuestionario que hipotéticamente mide cinco factores de estrategias identitarias: asumida, resistencia, simulación, atribuida y difusa. Acorde a la teoría de Castells (1998), se configuro cada estrategia por cogniciones (creencias), valores, prácticas y normas, se procedió a definir conceptualmente cada una de las estrategias identitarias. Los cinco factores exploran cómo se asumen los docentes en cuanto a las demandas de los nuevos modelos educativos y sus tipos de disposiciones, que van de la aceptación irreflexiva y automática a las demandas del modelo, el aparentar dar respuesta a las demandas mediante la simulación de roles, la confusión respecto a los nuevos paradigmas de la función docente que sustenta el modelo, la resistencia ante la nuevas demandas por considerar al modelo como una propuesta de carácter técnico o por negación ante prescripciones de la autoridad, y la posición reflexiva que mantiene una actitud propositiva.

Dicho cuestionario se estructuró con ítems que describían cada estrategia identitaria y con opciones de respuesta en escala Likert. Una vez diseñado el cuestionario, fue sometido a validez inter-jueces por expertos en teorías de identidad y expertos en construcción de instrumentos. Posteriormente, se realizó un estudio piloto. Se re-estructuró el cuestionario con los datos obtenidos de cada una de la fases citadas. El cuestionario quedó integrado por 29 reactivos.

\section{Procedimiento}

El estudio fue de tipo instrumental con el objetivo de obtener la validez y confiabilidad del cuestionario diseñado y verificar la prevalencia de cada estrategia en la población de estudio. La finalidad del estudio fue obtener la validez de constructo y consistencia interna del instrumento, para determinar su pertinencia para explorar las estrategias identitarias de los académicos universitarios de una universidad 
pública estatal y la presencia de indicadores de tensiones y/o crisis de identidad en estos. Los 228 académicos universitarios seleccionados por un muestreo por cuota, fueron entrevistados en el espacio institucional por los responsables de la investigación. Se les explicó en qué consistía el estudio y se les solicitó su participación voluntaria. El procesamiento y análisis de datos se realizó con el programa estadístico SPSS versión 22, se llevo a cabo el análisis de las frecuencias de cada reactivo y se analizó el sesgo y la curtosis. Por otra parte, se realizó el análisis de discriminación de reactivos por medio de la prueba t de student, y la consistencia interna de la prueba mediante el Alpha de Cronbach.

\section{Análisis de datos}

Los datos se procesaron mediante análisis factorial, confirmatorio de componentes principales por rotación varimax, para obtener la estructura factorial y se calculó el coeficiente Alpha de Cronbach para determinar la confiabilidad (consistencia interna). Para comprobar la adecuación del tamaño de la muestra se obtuvo el estadístico de KMO (Kaisen - Mayer Olkin). Se procedió a obtener la prueba de esfericidad de Barlett para comprobar la satisfacción de la estructura factorial, la congruencia con el grafico de sedimentación y el número de factores extraídos. Se obtuvieron estadísticas de tendencia central. Se conformó asimismo un modelo de acuerdo a la construcción hipotética, partió de la premisa de la presencia de cinco factores citados.

La estructura factorial hipotética se sustentó en un inicio en la propuesta teórica de estrategias identitarias de Camillieri (1990) y de Castells (1998) y, finalmente se enriqueció con base en la observación de las disposiciones de los docentes y su soporte en propuestas teóricas. Esto último permitió incluir las estrategias identitarias de simulación y difusa. Para la obtención de la estructura factorial del cuestionario de estrategias identitarias docentes, se consideraron los criterios de Hair y Tatham (1999) con respecto al número de participantes y el número de ítems. Se estimaron pesos factoriales por arriba de .40 . 


\section{Resultados}

\section{Descripción de la muestra}

Las condiciones laborales de los académicos que participaron en este estudio se describen a continuación. Con respecto al tipo de contratación se distribuyó de la siguiente forma: el 57.9\% (132) de los docentes fueronde asignatura y el $42.1 \%$ (96) de tiempo completo. En cuanto a la distribución por áreas de conocimiento el 6,6\% (15) provinieron del área de educación, el 32\% (73) pertenecieron a ciencias sociales, el $10.5 \%$ (24) se desarrollaron en artes y humanidades, el $12.7 \%$ (29) correspondieron a ciencias naturales y exactas, el 14.9. (34) a ingeniería y construcción; un 6.6 (15) laboraron en agronomía y veterinaria y un $16.7 \%$ (38) estaban adscritos a ciencias de la salud. En el grado académico, 28.5\% (65) contaron con nivel licenciatura; $37.3 \%$ (85) tuvieron maestría y 34.2\% (78) ostentaron nivel de formación doctoral.

\section{Validez y confiabilidad del cuestionario de estrategias identititarias de académicos universitarios}

La hipótesis de construcción del instrumento partió de la premisa de la presencia de cinco tipos de estrategias identitarias en los académicos universitarios. Esta hipótesis se comprobó, parcialmente, en virtud de que el primer factor aglutino a dos estrategias identitarias (resistencia y difusa) Los tres factores restantes fueron idénticos a los hipotéticos: simulación, atribuida y asumida (Ver Tabla 1). 
Diseño del cuestionario: Estrategias identitarias de académicos universitarios... / Zanatta et al.

\section{Tabla 1}

Matriz de la estructura factorial del cuestionario de estrategias identitarias de académicos universitarios

\begin{tabular}{|c|c|c|c|c|c|}
\hline \multicolumn{2}{|c|}{ NUMREACTVO } & \multicolumn{4}{|c|}{ PESO FACTORIAL } \\
\hline & Identidad resistencia difusa & D1 & D2 & D3 & $\mathrm{D} 4$ \\
\hline $\mathrm{R} 1$ & $\begin{array}{l}\text { El docente se resiste a desarrollar su práctica } \\
\text { académica en el modelo educativo institucional. }\end{array}$ & .817 & & & \\
\hline $\mathrm{R} 2$ & $\begin{array}{l}\text { La concepción de ser "docente" es poco clara en } \\
\text { el modelo educativo institucional. }\end{array}$ & .807 & & & \\
\hline R5 & $\begin{array}{l}\text { El docente considera que los documentos } \\
\text { rectores son poco claros. }\end{array}$ & .798 & & & \\
\hline R6 & $\begin{array}{l}\text { Las funciones docentes son poco claras en el } \\
\text { modelo educativo institucional }\end{array}$ & .794 & & & \\
\hline R7 & $\begin{array}{l}\text { El docente se resiste a las disposiciones que } \\
\text { marca el modelo educativo institucional }\end{array}$ & .774 & & & \\
\hline R11 & $\begin{array}{l}\text { Los docentes rechazan los documentos rectores } \\
\text { porque son imposiciones de corte "eficientista" }\end{array}$ & .756 & & & \\
\hline R12 & $\begin{array}{l}\text { El docente considera confuso el modelo } \\
\text { educativo institucional }\end{array}$ & .748 & & & \\
\hline R13 & $\begin{array}{l}\text { El docente rechaza el modelo educativo } \\
\text { institucional por ser una imposición } \\
\text { instrumental }\end{array}$ & .739 & & & \\
\hline R14 & $\begin{array}{l}\text { El docente rechaza el sustento teórico del } \\
\text { modelo educativo institucional }\end{array}$ & .714 & & & \\
\hline R18 & $\begin{array}{l}\text { El docente se resiste a las nuevas demandas de su } \\
\text { práctica educativa }\end{array}$ & .666 & & & \\
\hline R19 & $\begin{array}{l}\text { El docente experimenta desconcierto en su } \\
\text { quehacer respecto al modelo educativo }\end{array}$ & .624 & & & \\
\hline R20 & $\begin{array}{l}\text { El docente percibe confusa la misión que le dicta } \\
\text { el modelo educativo }\end{array}$ & .622 & & & \\
\hline R21 & $\begin{array}{l}\text { El docente percibe poco claras las cualidades que } \\
\text { les demanda el modelo educativo }\end{array}$ & .589 & & & \\
\hline R24 & $\begin{array}{l}\text { El docente debe oponerse a formar desde las } \\
\text { disposiciones del modelo educativo institucional }\end{array}$ & .54 & & & \\
\hline R27 & $\begin{array}{l}\text { La normatividad obstruye la libertad del trabajo } \\
\text { docente }\end{array}$ & .509 & & & \\
\hline R29 & $\begin{array}{l}\text { El docente desconoce los documentos que } \\
\text { describen el modelo educativo de la institución }\end{array}$ & .497 & & & \\
\hline
\end{tabular}




\begin{tabular}{|c|c|c|c|c|c|}
\hline \multicolumn{2}{|c|}{ NUMREACTVO } & \multicolumn{4}{|c|}{ PESO FACTORIAL } \\
\hline & Identidad atribuida & D1 & D2 & D3 & $\mathrm{D} 4$ \\
\hline R3 & $\begin{array}{l}\text { El docente asume sin objeción el modelo } \\
\text { educativo que la institución propone }\end{array}$ & & .77 & & \\
\hline R8 & $\begin{array}{l}\text { El docente debe ceñir su práctica al modelo } \\
\text { educativo institucional }\end{array}$ & & .704 & & \\
\hline $\mathrm{R} 15$ & $\begin{array}{l}\text { El docente debe aceptar el constructivismo que } \\
\text { sustenta al modelo educativo institucional. }\end{array}$ & & .675 & & \\
\hline $\mathrm{R} 22$ & $\begin{array}{l}\text { El docente debe acatar los documentos rectores } \\
\text { porque son realizados por expertos }\end{array}$ & & .636 & & \\
\hline $\mathrm{R} 25$ & $\begin{array}{l}\text { La función del docente es instrumentar el } \\
\text { modelo educativo que dicta la institución. }\end{array}$ & & .618 & & \\
\hline \multirow[t]{2}{*}{ R28 } & $\begin{array}{l}\text { El docente debe mantener disposición } \\
\text { incondicional a las demandas institucionales }\end{array}$ & & .587 & & \\
\hline & Identidad asumida & & & & \\
\hline R4 & $\begin{array}{l}\text { La misión del docente es reconstruir } \\
\text { críticamente la formación universitaria. }\end{array}$ & & & .78 & \\
\hline R9 & $\begin{array}{l}\text { El docente asume de manera crítica-propositiva } \\
\text { su profesión, integrándola a su proyecto de vida }\end{array}$ & & & .703 & \\
\hline R16 & $\begin{array}{l}\text { El docente critica propositivamente los } \\
\text { documentos rectores }\end{array}$ & & & .771 & \\
\hline \multirow[t]{2}{*}{ R23 } & $\begin{array}{l}\text { El docente reflexiona los valores institucionales } \\
\text { que asume }\end{array}$ & & & .538 & \\
\hline & Identidad Simulación & & & & \\
\hline $\mathrm{R} 10$ & $\begin{array}{l}\text { En apariencia, el docente, asume las reformas } \\
\text { curriculares }\end{array}$ & & & & .67 \\
\hline $\mathrm{R} 17$ & $\begin{array}{l}\text { El docente en apariencia asume el enfoque por } \\
\text { competencias }\end{array}$ & & & & .597 \\
\hline R26 & $\begin{array}{l}\text { El docente aparenta actuar conforme a los } \\
\text { valores institucionales }\end{array}$ & & & & .698 \\
\hline
\end{tabular}




\section{Análisis descriptivo del instrumento estrategias identitarias del docente}

El análisis descriptivo mostró que el Factor 1 (identidad de resistencia - difusa) implica una posición contraria a las disposiciones educativas (reactivos: R1, R7, R14, R18, R20, R21 y R24), originados por confusión de los referentes teórico-prácticos requeridos (reactivos: R2, R5, R6, R12, R19 y R29), con respecto al modelo educativo o por la consideración de este enfoque desde referentes teóricos antagónicos (reactivos R11, R13 y 27).

Este factor integró el mayor número de reactivos (16), En referencia a los otros factores. La conjugación de reactivos pertenecientes a los factores hipotéticos de identidad de resistencia e identidad difusa, guardo una proporción equitativa ( 8 reactivos para resistencia y 8 reactivos de difusión de la identidad docente). El factor original (identidad de resistencia) agrupó reactivos de resistencia a prácticas (R1), disposiciones (R7), demandas institucionales (R18) y rechazó a documentos rectores por ser de corte "eficientista" (R11), al modelo educativo por ser una imposición instrumental (R13) y al sustento teórico del modelo educativo (R14). Se opuso a disposiciones del modelo educativo (R24) y el considerar que la normatividad vigente obstruye la libertad de catedra (R27).

Con respecto al factor original: identidad difusa, se agruparon reactivos concernientes a la concepción del docente (R2), los documentos (R5) y las funciones docentes (R6); desconocer documentos del modelo educativo (R29) y prácticas docentes (R19); considerar confusa: misión (R20) y cualidades del docente (R21) y el modelo educativo institucional (R12). Dichos reactivos comprenden cogniciones, prácticas, valores y normas contrarios a la reforma educativa.

La media de (3.57) indica que la muestra se ubica arriba de la media teórica, lo que refleja una disposición antagónica ante las nuevas demandas de su práctica educativa por confusión en cuanto a lo que significa el ejercicio de la docencia respecto a referentes teóricos (cogniciones), valores, normas y prácticas que exige el nuevo modelo educativo o por poseer referentes contrarios a la demanda educativa. 
En cuanto al Factor 2 (Identidad atribuida), la media obtenida es de 3.42 , señalando que la muestra se encuentra por arriba de la media teórica y refleja que la práctica educativa, de estos docentes, se caracteriza por asumir las propuestas institucionales de manera automática sin un proceso de reflexión y, por tanto, sin la comprensión cabal de las demandas del modelo acerca de su función como formador. Sus funciones se ciñen rigurosamente a las demandas y políticas educativas (reactivos 3 y 8 , relativos a asumir sin objeción y ceñir su práctica al modelo educativo). Existe plena convicción en la autoridad, en la pertinencia de sus disposiciones y en el orden establecido (R22 y R25, relativos a acatar lo que realicen los expertos e instrumentar lo que dicte la institución). Se rige por valores de lealtad y obediencia a la autoridad (R28 y R15, mantener una disposición incondicional y aceptar lo que indique el modelo educativo).

En el factor 3 (Identidad Asumida), la media obtenida fue de 3.90. La media más alta por factor, obtenida por la población, pero con una agrupación de solo cuatro reactivos. Esta población se encuentra por arriba de la media teórica. Este factor constituye el polo positivo de la identidad. El sujeto se asume como docente desde sus referentes teóricos y materiales culturales de los que dispone (R4, reconstruir críticamente la formación universitaria), y construye una nueva identidad ante los cambios de paradigmas de su función docente (R9 y R23, reflexiona sobre los valores institucionales y asume crítica y propositivamente su profesión y R16 el docente critica propositivamente los documentos rectores). Los reactivos que configuran este factor comprenden cogniciones, prácticas y valores desde sus propios referentes, pero redefiniendo su posición respecto a su práctica educativa mediante una actitud reflexiva y propositiva.

Por último, en el Factor 4 (Identidad de simulación), la muestra obtuvo una media de 3.11 con la menor agrupación de reactivos (3), con una ubicación por arriba de la media teórica, reflejando que los académicos aparentan la identificación de valores (R26), la asunción de métodos, funciones y roles para con las demandas del modelo educativo (R17 aparenta asumir el enfoque de competencias y R10 aparenta asumir reformas curriculares). 
Diseño del cuestionario: Estrategias identitarias de académicos universitarios... / Zanatta et al.

\section{Tabla 2}

Descriptores de la población en la estructura factorial del instrumento de estrategias identitarias del docente

\begin{tabular}{lccccc}
\hline Factor & No. reactivos & $M$ & $D E$ & $M o$ & $M e$ \\
\hline 1. Resistencia - Difusa & 16 & 3.57 & .84 & 2.25 & 2.44 \\
2. Atribuida & 6 & 3.42 & .76 & 3.00 & 3.50 \\
3. Simulación & 3 & 3.11 & 1.02 & 3.67 & 3.33 \\
4. Asumida & 4 & 3.90 & .72 & 4.00 & 4.00 \\
\hline
\end{tabular}

\section{Estructura factorial acorde al modelo teórico propuesto}

En el estadístico de KMO (Kaisen - Mayer Olkin) se obtuvo un valor de .890 , lo que indica que el tamaño de la muestra fue adecuado, los resultados obtenidos en la prueba de esfericidad de Barlett $\left(X^{2}=5690.10\right.$ $p<.001)$ indican que la estructura factorial es satisfactoria.

Los cuatro factores explican el $43 \%$ de la varianza total (ver tabla 3) y cuenta con un Alpha de Cronbach de .94. El cuestionario quedó integrado por 29 ítems mediante extracción del análisis factorial. (Ver Tabla 3).

\section{Tabla 3}

Valores propios y varianzas del Cuestionario de estrategias identitarias de docentes

\begin{tabular}{lcccc}
\hline Factor & $\begin{array}{c}\text { Autovalores } \\
\text { iniciales }\end{array}$ & $\begin{array}{c}\% \text { de } \\
\text { varianza }\end{array}$ & $\begin{array}{c}\text { \% de varianza } \\
\text { acumulado }\end{array}$ & $\alpha$ \\
\hline 1. Resistencia-difusa & 12.84 & 24.77 & 24.77 & .94 \\
2. Atribuida & 4.81 & 8.07 & 32.84 & .78 \\
3. Simulación & 2.79 & 5.28 & 38.12 & .65 \\
4. Asumida & 1.59 & 8.49 & 43.01 & .67 \\
\hline
\end{tabular}




\section{Método de extracción: Análisis de Componentes principales}

\section{Validez de constructo discriminante}

Con respecto a la validez discriminante entre los factores que integran la estructura factorial, se halló una correlación negativa entre la identidad asumida (F3) para con la identidad resistencia - difusa (F1), con valores de $r=-.17$ y probabilidad .01 . Además, se halló una correlación negativa con el factor de identidad atribuida (F2) con valores de $r=-.30$ y probabilidad de .00 así como una correlación negativa con el factor de identidad de simulación (F4) con valores de $r=-.18$ y probabilidad de .010. Esto implica que los indicadores de la identidad asumida son contrarios con la identidad de resistencia difusa, la identidad de simulación y la identidad atribuida. Por otra parte, no se detectaron correlaciones entre los factores de identidad de resistenciadifusa, simulación y atribuida, mostrando la independencia entre las estrategias identitarias que exploran dichos factores.

\section{Discusión}

Los resultados mostraron que el instrumento para explorar el tipo de estrategias identitarias que asumen los académicos universitarios ante las reformas educativas, permite explorar cuatro tipos de identidades docentes que son conformados por disposiciones en cuanto a cogniciones, prácticas y valores. Dicha configuración es congruente con la teoría de Castells (1998).

Asimismo, la configuración de cada estrategia identitaria es también congruente con la propuesta de Harsch (1994), quien sostiene que la identidad profesional comprende la constitución de un yo-individual, un yo-teórico (cogniciones), un yo ético (valores) y un yo-empírico (prácticas). Mientras que lo teórico, lo ético y lo empírico configuran la identidad; el yo individual muestra el tipo de disposición de los docentes que permiten identificar cada tipo de estrategia identitaria. 
La estructura factorial se integró por factores derivados de la teoría de Castells (1998) y dos derivados de propuestas teóricas y de la observación. La identidad de simulación implica el aparentar la realización de prácticas conforme a las nuevas demandas del modelo educativo y la difusión de la identidad implica una confusión del ser docente en cuanto a los referentes teóricos, prácticas y valores. Conforme a la teoría de Erikson (1956) los cambio de valores y referentes que propician vacíos en la existencia generan una crisis de identidad, una confusión en cómo asumirse y proyectarse con un conjunto de disposiciones ante demandas de la vida, en este caso, de la vida laboral.

En el factor de resistencia - difusa, según se indica en la Tabla 2, se conjugaron indicadores de los factores teóricos hipotéticos de identidad de resistencia e identidad difusa. Los índices de identidad difusa reflejan prácticas de confusión en lo que se espera de él como docente en cuanto a su misión y las prácticas pedagógicas que requiere realizar en el nuevo modelo educativo condicionando una actitud contraria a las nuevas disposiciones. Los índices de resistencia muestran una disposición antagónica que, desde la postura de Castells (1998), se desarrolla por tener un marco de referencia contrario a la nueva demanda.

En la muestra de académicos que participaron en el presente estudio, prevalece la resistencia generada por la confusión de referentes cognitivos, éticos y prácticos que, probablemente, la instrumentación del modelo ha generado en el significado del ser docente. La resistencia, desde una posición crítica respecto al modelo, se muestra tan solo en tres indicadores: el docente rechaza el modelo educativo por ser una posición instrumental, el docente rechaza los documentos rectores por ser de corte eficientista y la normatividad vigente obstruye la libertad de cátedra.

Desde posturas críticas (López, 2003) considera que el modelo de competencias puede ser apreciada como una propuesta que favorece lo técnico e impone una visión instrumental de la educación. Asimismo, López (2003) sostiene que la construcción de la identidad de la profesión académica requiere de un proceso ético y no solo se sitúa en el nivel de la capacitación técnica. La formación de los formadores requiere de lograr sentido a su práctica laboral, sentido que se promueve a través 
de un proceso formativo que implique la reconstrucción de la identidad docente por medio de la reflexión de los nuevos materiales culturales desde sus propios referentes.

Aun cuando para Castells (1998) la posición reflexiva y crítica de la identidad de resistencia puede favorecer una identidad de proyecto (identidad asumida), la confluencia de este tipo de estrategia identitaria con la identidad difusa, en la muestra estudiada, refleja una prevalencia de indicadores de confusión y desconocimiento de las demandas del nuevo rol docente, en contraste con la posición del rechazo de la nueva propuesta por ser contraria a sus referentes teóricos y a sus convicciones respecto al sentido de la educación. Esto último implicaría la presencia de una identidad consolidada, posición que se muestra poco prevalente en los indicadores del factor de resistencia difusa.

En el polo negativo de la identidad, no solo se encontró el estado de confusión, también se detectó una apropiación por compromiso de la identidad que demanda la institución en el momento actual, a la que se denominó estrategia identitaria atribuida. Es considerada como parte del polo negativo, en tanto que es impuesta desde el exterior e instaurada sin un proceso de reflexión que permita el proceso de individuación y una disposición comprometida. Este tipo de estrategia, se caracteriza porque los actores sociales asumen las propuestas institucionales de manera automática sin un proceso de reflexión y sin la comprensión cabal de las demandas del modelo acerca de su función como formador. Existe plena convicción en la autoridad, en la pertinencia de sus disposiciones y el orden establecido, se rige por valores de lealtad y obediencia a la autoridad. Sus funciones se ciñen rigurosamente a las demandas y políticas educativas. Desde la postura de López (2003) la presencia de la obediencia automática de normas y convenciones, que no se han apropiado desde un proceso reflexivo, constituyen una falta de autonomía por un nivel precario en la dimensión ética que sustenta la construcción de la identidad.

La estrategia de simulación funciona como operación adaptativa y consiste en aparentar la identificación de valores, métodos, funciones y roles para con las demandas del modelo educativo, pero persisten 
prácticas anteriores. La disposición de los docentes que se muestra en este factor, es explicada desde la postura de Díaz-Barriga (2005), quien sostiene que los docentes pueden responder a la cosmovisión que representan el nuevo modelo educativo, pero no comparten una reforma que parte de otros presupuestos hacia la educación; por lo que se resisten a ello, y su resistencia se expresa en aparentar dar respuesta a las nuevas demandas. Para Andrade (2014), la asimilación de la identidad promovida por los grupos hegemónicos es favorecida por la socialización, pero también por motivos económicos y necesidad de asegurar la empleabilidad.

Por otra parte, en el polo positivo de la identidad, identidad de proyecto para Castells (1998), se encontró una estrategia identitaria asumida por el sujeto. En contraste con la atribuida desde el exterior, parte de un proceso de reflexión para lograr la individuación en el rol docente. De acuerdo a Castells (1998) permite a los actores, basándose en los materiales culturales de los que dispone, construir una nueva identidad y redefinir la posición de su práctica educativa. No existe una resistencia, ni una disposición automática, por el contrario, existe una actitud reflexiva y propositiva respecto a la práctica, la normatividad y los valores, favoreciendo una actitud comprometida y disposición a su misión como docente.

La identidad asumida es la condición de posibilidad de una postura crítica ante nuevas demandas a la función docente que permite lograr consistencia en referentes teórico, práctico, éticos y es la base de una actuación comprometida del docente. La importancia de lograr una identidad asumida en los docentes radica en que favorece una práctica docente comprometida. Caballero (2014), sostiene que las creencias del profesorado acerca de la docencia condicionan en gran medida su estilo de enseñanza. El autor afirma que la transformación de las creencias acera de la enseńanza, sus modos de hacer y de entender el aprendizaje no es una labor que pueda programarse ni conseguirse de un día para otro.

Los valores propios y varianza reportados por el instrumento, la coherencia teórica del contenido de cada factor y de la estructura 
factorial para con la hipótesis de construcción, corroboran la validez de constructo y consistencia interna del cuestionario diseñado, así como su sensibilidad para explorar estrategias identitarias en académicos universitarios.

La varianza explicada por cada factor muestra la prevalencia de estrategias de identidad de resistencia-difusa y de simulación, lo que indica la presencia de crisis y tensiones en el desarrollo de estrategias identitarias de los académicos ante las demandas del modelo educativo. Crisis y tensiones en la identidad que son reflejo de la necesidad de un proceso formativo desde lo ético que favorezca la construcción de un "Ethos docente" y, con ello, incida en el desarrollo de un conjunto disposicional de los docentes de formadores de profesionales que tendrán que atender demandas sociales de la actualidad.

Acorde a la propuesta de Caballero (2013), la calidad de la docencia requiere de impulsar procesos formativos docentes que favorezcan la construcción de sentidos de las nuevas exigencias al profesorado por medio de procesos formativos. Asimismo, Muñoz y Arvayo (2015) en atención a los elementos que configuran la identidad, proponen formación que fortalezca la identidad docente como competencia de síntesis, atendiendo el desarrollo de nuevas competencias significadas por procesos de reflexión y contextualización. En congruencia, Hodelín y Fuentes (2014), proponen una formación universitaria reflexiva, participativa e interactiva que favorezca la construcción de lo cognitivo, práctico y ético.

La construcción de un "Ethos" docente requiere de nuevos dispositivos formativos sustentados en dichos procesos reflexivos, participativos en interacción con el contexto, que favorezcan una cultura en torno a las nuevas demandas del rol docente. Caballero y Bolívar (2015) advierten que la modificación de creencias, prácticas y valores implicados en la construcción de estilos identitarios que favorezcan la docencia universitaria no es tarea sencilla y requiere un replanteamiento de los sistemas de formación y evaluación, así como nuevos dispositivos y contextos que posibiliten favorecer las disposiciones hacia los nuevos paradigmas y los nuevos roles. 
Diseño del cuestionario: Estrategias identitarias de académicos universitarios... / Zanatta et al.

\section{Referencias}

Andrade, J.A. (2014). Identidad profesional y mundo contemporáneo: Reflexiones desde un resumen de un caso. Athenea Digital, $14(2), 117-145$

Caballero, K. (2013). Nivel de satisfacción del profesorado universitario hacia los sistemas de evaluación. Revista de Educación, 360, 483-508.

Caballero, K. (2014).El profesor universitario y su identidad profesional. Revista de docencia universitaria. REDU, 13(1), enero-abril 2015, 57-77

Caballero, K. y Bolívar A. (2015). El profesorado universitario como docente: hacia una identidad profesional que integre docencia e investigación. REDU - Revista de Docencia Universitaria, 13(1), 57-77. Publicado en http://www.red-u.net

Camillieri, C. (1990). Estrategias identitarias [Traducción]. Paris: PUF.

Castells, M. (1998). La era de la información: economía, sociedad y cultura. El poder de la identidad. Vol. 2. Madrid: Alianza.

Díaz-Barriga, A. (2005). El profesor de educación superior frente a las demandas de los nuevos debates educativos. Perfiles Educativos, 27(108), 9-30.

Ducoing, W. (2005). Sujetos, actores y procesos de formación: la investigación educativa en México. México: Consejo Mexicano de Investigación Educativa.

Erikson, E. (1956). El problema de la identidad del ego [Traducción]. Journal of The American Psychoanilytic Association, 4, 56-121. https://doi.org/10.1177/000306515600400104

Hair, A. y Tatham, B. (1999). Análisis Multivariante (1 ${ }^{a}$ Ed.). España: Prentice Hall Iberia S.R.L., Colección.

Harsch, C. (1994). Identidad del psicólogo. México: Alambra Mexicana. Hodelín T. y Fuentes P. (2014) Formación de profesionales universitarios. Un desafío bioético. Medisan [serie en Internet]. Recuperado el 4 de mayo de 2016, de: http://bvs.sld.cu/revistas/ san/vol 8_1_04/san12104.htm 
López, Z. R. (2003). Ética de la profesión académica en la época global. En A. Hirsch (Eds), Ética profesional e identidad institucional (pp. 245-265). México: UAS.

Muñoz, O. F. y Arvayo, M. K. (2015, noviembre). Identidad profesional: ¿Qué significa ser docente? European Scientific Journal 11 (32) e -ISSN 1857- 743197

Rosas, P.C.E. (2015). Relación de los factores profesionales, académicos y las competencias pedagógicas del docente de la Universidad Católica de Trujillo. Revista de Investigación y Cultura, 4(1)6, 97-100.

Yurén, M. T. (2005). Ethos y autoformación en los dispositivos de Formación de docentes. En M. T. Yurén (Ed.), Ethos y autoformación del docente: análisis de dispositivos de formación de profesores (pp. 19-45). Barcelona: Ediciones Pomares.

Recibido: 13 de mayo, 2015

Revisado: 6 de abril, 2017

Aceptado: 18 de abril, 2017 